\title{
IMPACTO SOCIOCULTURAL DE LA ACTIVIDAD MERCADOLÓGICA EN EL SER HUMANO Y LA SOCIEDAD
}

\author{
SOCIOCULTURAL IMPACT OF MARKETING ACTIVITY \\ IN THE HUMAN BEING AND SOCIETY
}

Ricardo Arturo Valdez Cornejo

Glo-Val. Consultoría y Asesoría Empresarial, Arequipa, Perú

\section{Resumen:}

El presente trabajo trata acerca del impacto social y cultural de la actividad mercadológica empresarial, tomando como eje fundamental la actuación de las organizaciones y la sociedad con la que entran en contacto, la cual presenta cambios que pudieran resultar positivos o negativos, vistos desde un enfoque amplio y objetivo. Por otro lado, la sociedad también influye sobre la forma cómo las empresas llevan a cabo sus actividades de carácter mercadológico. Por tanto, existe una clara sinergia entre empresa y sociedad, la cual debiera ser aprovechada para generar maximización de valor compartido en un contexto de responsabilidad social corporativa y plena conducta ética.

Palabras clave: Impacto, marketing, sociedad, cultura, ser humano, responsabilidad social corporativa.

\section{Summary:}

The purpose of this work is focused on the social and cultural impact of the matketing business, taking as fundamental edge the presence of organizations and the society they are in contact with, which presents changes that could be positive or negative, seen from a wide and objective approach. Otherwise, society also influences on the way the companies carry through their activities of marketing character. Therefore, 
there is a clear synergy between business and society, which should be exploited to generate maximizing shared value in a context of corporate social responsibility and full ethical conduct.

Key words: Impact, marketing, society, culture, human being, corporate social responsibility.

"Existe una gran diferencia entre ganar dinero para ti mismo y crear riqueza para los demás". "Poder y riqueza no son dos de mis principales apuestas".

Ratan Tata, Empresario Indio Presidente del Grupo Económico Tata (India)

\section{Introducción}

Toda ciencia, arte, oficio o saber humano impacta indefectiblemente sobre la cultura y la sociedad, sin embargo, este impacto puede ser positivo o negativo. De allí la importancia de considerar como stakeholders a todos los grupos de interés no solamente beneficiados, sino también, perjudicados por la actividad de las organizaciones empresariales y no empresariales. Dentro de estas actividades humanas, artes y ciencias, se encuentra el marketing, al cual se va a analizar en este artículo. En ese sentido, pretendemos responder las siguientes interrogantes:

¿El marketing, al ser una actividad de carácter social, impacta sobre la sociedad y el entorno cultural? Asimismo, ¿Este impacto puede ser positivo o negativo?, $¿$ Las empresas deben promover cambios positivos sobre las sociedades con las que interactúan, a través de programas de responsabilidad social bajo un marco de actuación ética?, ¿Desde una óptica de responsabilidad social corporativa, las empresas deben limitarse a solucionar aspectos netamente operativos como disminuir el impacto medio ambiental propio de algunas actividades; o debe contribuir a la formación de conciencia apoyada en esfuerzos inclusivos y bajo un marco ético?, ¿Todo cambio social o cultural debe generar progreso, tanto en lo referente a calidad de vida, como a nivel de vida? Asimismo, ¿El marketing puede ser agente de cambio activo en este sentido?

\section{Desarrollo}

Se debe recordar que el marketing es una actividad de carácter social y administrativo (Kotler y Armstrong, 2013), por lo que su impacto suele ser mayor al de otras actividades, como las relacionadas con ciencias exactas, físicas y formales. Asimismo, por esta razón, es importante recalcar que los cuidados que se debe tener son mayores a los que suele haber en el marco de otras actividades como las mencionadas; ya que el impacto nocivo que se puede originar podría ser extremadamente fuerte. Por fortuna, el impacto también puede ser positivo... muy positivo. 
De hecho, a través del marketing se puede cambiar ideas paradigmáticas, puntos de vista, inclinaciones, preferencias... E incluso, esquemas culturales complejos de forma completa. $\mathrm{O}$, también, se puede cambiar hábitos y conductas, como sucedió en el caso del ingreso de los centros comerciales bajo el concepto de mall en la ciudad de Arequipa.

Desde una óptica conservadora, se podría considerar que las conductas sociales, culturales y de compra de los ciudadanos de esta ciudad sureña eran de corte tradicional y que, por tanto, la instalación de un centro comercial con las características de gran superficie no daría los frutos esperados por los inversionistas. Cuando el centro comercial abrió sus puertas durante el año 2001, la expectativa se convirtió en una realidad que rebasó los límites de toda planificación previa realizada por el grupo económico que apostó por esta nueva forma de hacer comercio en una zona en la que se había estado habituado a mirar estas actividades desde una óptica completamente diferente.

Lo mejor de todo, cuando ocurrió este acontecimiento empresarial y social es que, contrariamente a lo que se pensaba, toda la actividad de intercambio de bienes por dinero se dinamizó generando oportunidades nuevas para gran parte de los empresarios formales e informales de los rubros comerciales, en virtud que se celebró una importante apertura de mente, se vencieron ideas paradigmáticas que en nada beneficiaban al desarrollo, se generaron nuevos puestos de trabajo y la ciudad empezó su franco e imparable proceso de desarrollo sostenible hasta la fecha presente, aunque no necesariamente sustentable y posiblemente no sostenible en un futuro como modelo tal cual se ha concebido y se ejecuta en la actualidad debido a que en gran medida esta economía se basa en actividades extractivas que no generan mayor valor agregado a los productos tanto para un mercado interno como para las exportaciones, como es el caso de la minería, ya que aún no se logra superar los niveles de exportaciones tradicionales en favor de las no tradicionales.

Asimismo, el impacto medioambiental producido por la contaminación derivada de diversas actividades industriales y la falta de controles al respecto, unido a un excesivo crecimiento descontrolado del parque automotor y de las zonas residenciales en detrimento de la campiña hacen que posiblemente la sostenibilidad sea una utopía más que una realidad, tomando en cuenta que el índice de desarrollo humano no solamente debe medirse a través de bienestar económico o nivel de vida.

Es un hecho que el PBI de Arequipa se ha visto incrementado. Éste creció en un 108,2 \% en el período 2001 a 2012, tomando como año base 1994, lo cual fue superior al promedio nacional (96,9\%) (INEI, 2015). El período mencionado es importante porque justamente el año 2001 es en el que se inicia este proceso de cambios en aspectos diversos como la transformación en las tendencias de consumo, unido a otros factores que se consolidan hacia el año 2012.

Por otro lado, la pobreza disminuyó de $24,9 \%$ a 19,6 \% entre el período 2005 al 2010, aunque lo cierto es que la pobreza extrema se mantuvo en 3,8 \% considerando 
ambos años de referencia, con algunas fluctuaciones entre éstos (Observatorio Socio Económico Laboral MINTRA, 2015). Se observa entonces, que la disminución de la pobreza no ha sido significativa y que la pobreza extrema al mantenerse en niveles similares, indica que el aparente progreso de la sociedad no es igualitario para todos, lo cual debe llamarnos a una seria reflexión.

A esto se suma la saturación de vehículos motorizados, a veces innecesarios, con el consiguiente tráfico en crecimiento, que no soportan las estrechas vías céntricas de una urbe que es patrimonio cultural de la humanidad como justo tributo de la UNESCO a un pueblo pujante que generó un estilo arquitectónico único en el mundo como es el barroco mestizo Sur Peruano, maravillosa fusión de los aportes andino y europeo. De hecho, la ciudad de Arequipa recibió el importante nombramiento en noviembre del año 20oo. Por otro lado, se ha perdido parte de la identidad, así como la tranquilidad propia de una ciudad provinciana que se fue convirtiendo en metrópoli cosmopolita. El resultado final, es que se generó una vorágine imparable de consumismo desmedido y en ocasiones, atentatoria contra la dignidad humana.

Precisamente, todo lo expresado, genera la base de este análisis que trata acerca de los cambios positivos y negativos en lo social y cultural, generados por la actividad mercadológica.

Los impactos culturales:

- Disolución de la identidad. No consideramos sea pérdida de identidad.
- Apertura de mente.

- Mayor conciencia de consumo, desde una óptica de cliente.

- Mayor conciencia de respeto hacia los derechos de los consumidores.

Los impactos sociales:

- Mayores presiones de los grupos sociales.

- Mayor stress generado por el deseo consumista.

- Mayores frustraciones al no alcanzar metas que antes no existían o que no resultaban visibles.

- Consumismo desmedido.

Acompañando todos estos cambios, se generan algunas reacciones adversas de las poblaciones que entran en contacto con las organizaciones empresariales, las cuales no siempre son aceptadas de buen grado. Una forma interesante y efectiva de aceptación social en medios en los que la interacción entre empresa y población no siempre es positiva o es difícil, se evidencia en las diferentes acciones de responsabilidad social corporativa que debieran acompañar toda actividad empresarial, no porque la normativa legal lo exija, sino como auto imposición de carácter moral.

Asimismo, es posible utilizar los esfuerzos mercadológicos en direcciones diferentes a la meramente comercial, como en el caso del marketing social, que como concepto se puede basar en la siguiente afirmación: "El 
marketing social es el diseño, implementación y control de programas, dirigidos a incitar la aceptación de ideas sociales, mediante la inclusión de factores como la planeación del producto, precio, comunicación, distribución e investigación de mercados" (Kotler y Zaltman, citados por Pérez, 2004, p. 3).

De hecho, a través del marketing se puede apoyar causas diversas, las cuales encuadran perfectamente dentro de la responsabilidad social corporativa, la cual, además, debe ser parte integrante e inseparable de un proceso de planificación estratégica.

Existe una amplia gama de experiencias con sus respectivos resultados a escalas monumentales en un número importante de corporaciones que han utilizado el marketing para dar a conocer programas de responsabilidad social, aunque en muchas ocasiones puede resultar controversial la excesiva "publicidad social" que no va de la mano con la ponderada responsabilidad social, ya que la primera es exagerada respecto a la segunda. Asimismo, en ocasiones, el trabajo de las fundaciones de diversas corporaciones es tomada como filantropía pura, cuando en realidad son parte de un negocio sumamente rentable basado en la deducción de impuestos en el país en el que se ejecuten sus actividades, a cambio de realizar algunas acciones sociales, generalmente ligadas a alimentación, salud, educación o infraestructura.

Por otro lado, desde un ángulo observado a partir del punto inverso, el cual corresponde a la ciudadanía en general, se puede afirmar que no siempre las corporaciones impactan positivamente. Lo que se debe hacer es buscar consenso en base al diálogo abierto en el cual intervengan no solamente las corporaciones y la sociedad civil organizada, sino también representantes del poder gubernamental, como es el caso de las consultas previas que encuadran dentro de un concepto sano de cabildeo bien entendido y no de lobby llevado a cabo en esferas de poder económico y político de muy alto nivel, destinados a encubrir falencias irreconciliables y atentatorias contra la sociedad, en favor de unos cuantos grupos de poder económico.

También, los organismos sin fines de lucro pueden llevar a cabo sus acciones sociales apoyadas en grupos empresariales importantes, sin embargo:

La aceptación de ayuda económica de empresas con un objeto social cuestionable éticamente (tabaco, bebidas alcohólicas, fabricación de armamento...) por parte de organizaciones no gubernamentales (ONG) que suscriben un ideario muy determinado constituye un problema de plena actualidad gracias al auge del denominado marketing con causa. (García, 2000, p. 107)

Respecto a este punto, es conveniente anotar que algunas acciones sociales se pueden ver salpicadas de controversia debido a lo mencionado en el párrafo precedente, lo cual podría ser contraproducente e ir en detrimento de la imagen de las organizaciones con fines sociales e incluso, socavar hasta sus bases más elementales, alcanzando una debilitada filosofía organizacional asentada sobre misión, visión y valores no siempre bien planteados. 
Por ende, no basta con planificar acciones de responsabilidad social corporativa, sino analizar muy bien la procedencia de todo apoyo a las diversas causas sociales. Todo esfuerzo de marketing social, del cual se espera un importante impacto sociocultural, no es bueno o malo; así como efectivo o inefectivo per se, sino que va a depender de una diversidad de factores, variables o condiciones, como las mencionadas.

Más allá del impacto mercadológico y de imagen institucional que se pueda alcanzar, se deberá pensar en el impacto realmente útil y trascendente para la persona, la sociedad y la cultura.

Por otro lado, los esfuerzos del marketing social -y del comercial también- deben considerar siempre el desarrollo humano como eje central, diferenciando claramente lo que es nivel de vida de calidad de vida, tomado en consideración que:

Cuando hablamos de desarrollo humano y de reducción de la pobreza, no debemos referirnos al consumo desenfrenado de mercancías (...), sino al hecho de que todos los seres humanos puedan satisfacer sus necesidades básicas de alimentación, salud, vivienda, educación, (...), así como de disponer de tiempo suficiente para gozar de la cultura y de las artes, tener relaciones sociales enriquecedoras, hacer realidad nuestras vocaciones legítimas en cualquier ámbito que elijamos y, asimismo, tener tiempo libre para el descanso. Se trata de una concepción de la riqueza humana, y por consiguiente de la pobreza, que va mucho más allá de la esfera de la economía y de su evaluación monetaria o mercantil. (Angulo, 2010, p. 33)
Los aspectos mencionados en la cita en el párrafo anterior otorgan la base para una reflexión de suma importancia y que toma verdadera relevancia en tiempos actuales en los que la sociedad y la cultura se han visto alteradas por la vida de modernidad en la que intenta desarrollarse el ser humano; aunque este desarrollo lo ha conducido a una especie de tolvanera que parece no tener un fin deseable o, por lo menos, adecuado.

Se mencionaba que la calidad de vida se ha perdido en pro del nivel de vida, dos conceptos en nada parecidos y que en ocasiones son totalmente contrapuestos en la práctica. El nivel de vida hace referencia al estatus alcanzado por una persona inmersa en una colectividad, tanto en el aspecto económico, como en el cultural y social. La calidad de vida hace referencia al bienestar alcanzado por el ser humano en estado de equilibrio, y en armonía con la naturaleza, otras personas y el propio ser interior; considerando, incluso aspectos de simbiosis enriquecedora y homeostasis plena.

Por tanto, la calidad de vida debe abarcar aspectos tales como plena espiritualidad, felicidad, equilibrio físico y mental, entre otros; indicadores y variables todas conducentes a la autorrealización del ser.

Asimismo, definitivamente, los aspectos socioculturales marcan las tendencias y conductas de consumo entre los diferentes grupos de personas que conforman los mercados objetivos. Para entender mejor en qué consisten estos aspectos, se puede recurrir a la siguiente cita respecto al tema en mención: 
...los individuos se encuentran en constante movimiento y tienden a la segmentación o agrupación de manera natural, es decir, los individuos se agrupan en colonias y viviendas con base en perfiles sociales, económicos y culturales muy similares, lo que permite su ubicación y clasificación temporal. (Pérez, 2004, p. 19)

Todo esto, a veces sin darnos cuenta, facilita a las empresas la toma de decisiones en base a una adecuada segmentación de tipo psicográfica o por estilos de vida (Arellano, 2001), o, en su defecto, a una acertada segmentación demográfica.

La segmentación mencionada cada vez se ha tornado más compleja por las propias exigencias del mercado y la sociedad. En un inicio, las empresas se limitaban a llevar a cabo una segmentación geográfica elemental e incluso, precaria; posteriormente, las empresas se fijaron más en la segmentación demográfica y conductual, en un marco de mayor competitividad propia del marketing orientado a los clientes.

Hoy en día, en un entorno de marketing 3.o, en el cual, la responsabilidad social es eje fundamental; la segmentación psicográfica toma dimensiones realmente vitales para las organizaciones empresariales. Esta segmentación por estilos de vida es mucho más compleja y completa, lo cual conduce a mejores resultados, más aún en una sociedad cambiante y en franco desarrollo, ya que otorga mayores posibilidades de desarrollo a las mismas empresas, al no estar circunscritas a factores ya conocidos y muchas veces manejados de forma demasiado genérica, como son la edad, sexo, nivel socio económico o lugar de residencia.
Los estilos de vida tocan dimensiones mucho más profundas, al enfocarse en grupos de personas que tienen inclinaciones, usos, costumbres, valores y creencias similares (Arellano, 2001); lo cual crea mayores vínculos emocionales fundamentales para crear lealtad de marca y lograr, como paso previo, identificación de un segmento o nicho de mercado que comparte características en común y que, por tanto, se ve impactado social y culturalmente por el marketing de manera muy similar entre todos sus componentes humanos.

Por otro lado, es sumamente importante recalcar la función de la empresa como agente de cambio social y cultural. Al respecto, es útil señalar lo siguiente:

Agente de cambio: es la persona física y/o moral responsable del programa social. Todo programa social debe tener de manera explícita el nombre de las instituciones responsables del programa social. Es importante construir una red de instituciones involucradas de manera directa e indirecta en los programas sociales. Estas instituciones pueden ser del sector gubernamental, privado o del Tercer Sector. El agente de cambio debe ser el ente responsable de la detección de las necesidades sociales y de la satisfacción de las mismas con apoyo de recursos proporcionados por los diferentes grupos que intervienen en el programa social. La responsabilidad de la administración de los programas sociales depende directamente del agente de cambio y de la red oportuna y adecuada de cooperación que construya para implicar a personas físicas, morales y dependencias gubernamentales en apoyos tangibles y mesurables del programa social. (Pérez, 2004, p. 156) 
Sin embargo, es conveniente aclarar que las acciones de estos agentes de cambio, cualquiera sea el sector en el que se desenvuelvan, no pueden ser llevadas a cabo de manera casual o al azar, lo que significa que no están exentas de una adecuada planificación estratégica, ya que:

El agente de cambio tiene que diseñar planes de marketing bien diferenciados para cada uno de los distintos grupos involucrados, por lo que la labor de marketing para una organización sin fin de lucro se hace más extensa y laboriosa que para el sector privado, ya que sin apoyo no se contará con los recursos necesarios para la operación y sin población objetivo no existirá el programa social. Se debe diseñar un plan integral de marketing con bases sólidas que se estipulen en la misión, en la visión, en los objetivos y en las estrategias generales que conformarán los planes específicos para cada uno de los grupos incorporados. Se debe involucrar a cada grupo en los diferentes planes tácticos con las medidas de desempeño y resultados sociales respectivos. (Pérez, 2004, p. 158)

Asimismo, es interesante observar que ciertos acontecimientos que se suceden en un mundo globalizado impactan fuertemente en la forma cómo se conciben y desarrollan las actividades mercadológicas. Tal es el caso del fenómeno migratorio que ha impactado fuertemente en los hábitos de consumo de las personas, aunque no necesariamente el impacto haya sido positivo. Es conveniente, recordar que:

La migración de los residentes rurales a las áreas urbanas con inadecuada infraestructura de apoyo y pocas oportunidades de empleo crea problemas adicionales. La caída de los precios de los productos agrícolas continúa deteriorando las condiciones de vida en las áreas rurales de ALC, lo que fuerza a las personas a migrar hacia las grandes ciudades. En Centroamérica los campesinos constantemente abandonan las adormecidas comunidades rurales y se dirigen a las grandes urbes en busca de oportunidades. De manera inevitable terminan viviendo en casuchas de lámina y madera construidas a lo largo de las vías del ferrocarril o a las orillas de los ríos en lugares como La Chacra, San Salvador, o en zonas montañosas alrededor de Tegucigalpa, la capital de Honduras. En Centroamérica, las guerras civiles en Guatemala y Nicaragua provocaron grandes migraciones. Lo mismo ocurre periódicamente a causa de desastres naturales como huracanes, terremotos y sequías recurrentes. (Kotler, Gertner, Rein \& Haider, 2007, p. 30)

Lo expresado en párrafo precedente indica que a lo largo de la historia pudieran ocurrir gran cantidad de eventos que impacten negativamente sobre públicos vulnerables. Tal es el caso de personas que motivadas por la esperanza de una vida mejor y atraídas por fuertes programas de comunicación mercadológica, migran en busca de oportunidades que supuestamente mejorarán su calidad de vida, pero lo único que consiguen es intentar ascender muy ligeramente en cuanto a nivel de vida en detrimento de la calidad de vida, la cual podría haber sido claramente superior en su estado inicial, incluso a pesar de las condiciones inequitativas de acceso a algunos servicios propios de la modernidad o en situaciones de conflicto social.

Entonces, no solamente basta con crear un atractivo para las personas vistas como 
futuros clientes, sino en otorgar verdaderas soluciones integrales que conlleven en la práctica a un desarrollo humano integral. Asimismo, para entender adecuadamente el impacto del que se habla en el presente trabajo, es necesario indicar que se hace necesaria una investigación intensa de carácter cualitativo. Al respecto, es importante anotar que se ha considerado durante mucho tiempo a la investigación cuantitativa como la de mayor validez y exactitud, sin embargo, es conveniente recalcar que la investigación cualitativa también aporta importantes ideas para la gestión empresarial y en general, organizacional y social. Una herramienta interesante, en este sentido, es la investigación etnográfica.

La investigación etnográfica es el estudio del comportamiento humano en su contexto natural, e implica la observación de la conducta y el entorno, junto con entrevistas en profundidad. En ocasiones también se utilizan grabaciones de audio y video. De esta manera, se combinan las técnicas de interrogación y de observación para entender el comportamiento de los consumidores. (Malhotra, 20o8, p.210)

Las percepciones de las personas que conforman cada nación asentada sobre la faz de la Tierra son muy diversas y repercuten en el consumo y en la valoración de los productos y / o servicios de manera muy diferente en cada lugar.

Las repercusiones de la cultura son tan naturales y automáticas que su influencia en el comportamiento generalmente se da por sentada. Por ejemplo, cuando los investigadores del consumo preguntan a las personas por qué hacen ciertas cosas, a menudo éstas contestan: "porque es lo correcto”. Esta respuesta, que pareciera superficial, refleja en parte la influencia tan arraigada de la cultura en nuestro comportamiento. A menudo, solamente nos volvemos conscientes de cómo la cultura ha moldeado nuestro comportamiento cuando nos vemos expuestos a personas con diferentes costumbres o valores culturales (como cuando visitamos una región o un país diferente). Así pues, una verdadera apreciación de la influencia que la cultura tiene en nuestra vida diaria requiere cierto conocimiento de, por lo menos, otra sociedad con características culturales distintas. Por ejemplo, para entender que cepillarse los dientes dos veces al día con un dentífrico con sabor es un fenómeno cultural, se requiere algo de conciencia de que los miembros de otra sociedad no se cepillan los dientes o lo hacen de una manera muy distinta a la de nuestra sociedad. (Schiffman, 2010, p. 349)

Todo esto ocurre porque los individuos se ven reflejados en su sociedad; asimismo, son el reflejo de ésta. Es por ello, que resulta de utilidad considerar que:

Los consumidores se ven a sí mismos en el contexto de su cultura y reaccionan a su ambiente con base en el esquema cultural que llevan consigo al enfrentarse a esa experiencia. Cada individuo percibe el mundo a través de su propia lente cultural. (Ueltschy \& Krampf citados por Schiffman, 2010, p. 349)

De hecho, no siempre las acciones consideradas como correctas por las personas en algunas sociedades son las más aceptadas en sentido global ni ética ni prácticamente hablando. Mucho va a depender de diversos puntos de vista. 
El marketing, definitivamente, aprovecha en gran medida las variables culturales y sociales y debe hacerlo de esta forma, ya que, de lo contrario, es posible que las empresas con sus respectivos productos o servicios no tengan sostenibilidad ni sustentabilidad. También es conveniente anotar que en diversas oportunidades, los clientes aprenden en un entorno cada vez más competitivo y exploran nuevas dimensiones, obligando, a su vez a las empresas, a ser más competitivas. Tal es el caso de la "cultura del reclamo" para recibir el servicio justo o el producto de mayor utilidad o calidad percibida. Cada vez son más los clientes que buscan sentirse complacidos y no solamente satisfechos. Para esto, se debe recordar que:

El reclamo es la manifestación de descontento o insatisfacción que expresa un consumidor ante una empresa, sea de forma oral o escrita, con respecto a un bien o servicio. Al reclamar, el consumidor espera una compensación: ser indemnizado o que le cambien el producto, por ejemplo. La gestión de reclamos es un servicio posventa que las empresas pueden utilizar para desarrollar una oferta integral y alcanzar una ventaja competitiva. Es un proceso que implica recibir, investigar, resolver, cerrar y prevenir reclamos, y cuyo objetivo es satisfacer al cliente que reclama. (Puente \& López, 2007, p. 52)

De lo anterior, se desprende como idea aplicable que una forma de hacerse respetar como sociedad y cultura es saber aplicar la cultura del reclamo, la cual no hace sino demostrar la capacidad de autodefensa de una nación y, de paso, enseña permanentemente a las empresas acerca de lo que es practicar un buen servicio al cliente.
Asimismo, también en el marco del mutuo impacto cultural, las empresas se han visto obligadas a cambiar ciertos mensajes comunicacionales y la forma de transmitirlos, tanto por presiones culturales como sociales de naciones que cada vez se respetan más. Por ejemplo, en el caso de las publicidades televisivas de cervezas en Perú, quedó muy en claro que el poblador promedio ya se sentía incómodo por la utilización desmedida, así como de manera inadecuada y denigrante del cuerpo femenino. Esto se puso de manifiesto entre otras cosas, en el decreto legislativo 691 acerca de las normas de la publicidad en defensa del consumidor, de cuyo texto se destaca el artículo 3 que menciona expresamente: "Ningún anuncio debe favorecer o estimular cualquier clase de ofensa o discriminación racial, sexual, social, política o religiosa" (DEMUS, 2015, p. 12), y que, además, emana de la misma constitución política del Perú.

De hecho, se pone de manifiesto una vez más, que es lo que la mercadotecnia no debe hacer, tomando en cuenta consideraciones éticas (Valdez, 2015, p. 54):

- Publicidad cargada y sobre cargada de mensajes con alto contenido sexual implícito o explícito, violencia consentida y disfrazada; así como incitaciones inapropiadas.

- Publicidad inadecuada y agresiva dirigida al público más frágil y vulnerable, como son los niños e infantes.

- Publicidad engañosa, mostrando bondades inexistentes de los productos, recurriendo a información inexacta adrede. 
- Publicidad cargada de mensajes subliminales que pretenden atacar la vulnerabilidad del ser humano de la forma más salvaje e irracional.

Bajo este marco, el enfoque de las publicidades de cerveza cambió y las empresas productoras de esta "droga social" se enfocaron más hacia la amistad, el orgullo nacional e incluso hacia la propia idoneidad del producto en el caso de las cervezas tipo "Premium". Sin embargo, alguna marca continuó emitiendo mensajes atentatorios contra públicos vulnerables al transmitir sus ideas cargadas de palabras de doble sentido y utilizando monserga de manera sumamente exagerada. Lo interesante fue observar que esta marca no tuvo la aceptación esperada, lo cual también podría denotar un importante cambio cultural en Perú. Tal fue el caso de cerveza Brahma, la cual ingresa al país con expectativas muy superiores a los resultados generados.

Queda claro que las empresas deben ser cuidadosas en los mensajes comunicacionales emitidos, como parte de planes de marketing que a su vez se ven ligados a planes de negocios estratégicos integrales. Asimismo, una sociedad cada vez más instruida será cada vez más exigente. Otro aspecto sumamente importante respecto al impacto cultural es el enorme avance que se ha logrado en muchos stakeholders o grupos de interés a diversos niveles, acerca del cuidado de la ecología. Las ideas de aplicar las tres "Rs": Reducir, reutilizar y reciclar; cobran real importancia en tiempos modernos en los que la naturaleza se encuentra excesivamente fatigada y en muchos casos, agonizante. De hecho, de las tres acciones posibles mencionadas, la de recudir el consumo es la más efectiva de todas. Este aspecto es uno de los que falta desarrollar en la gran mayoría de las campañas mercadológicas, ya que las empresas continúan lanzando mensajes consumistas en cada comunicación que realizan. Este, quizás, sea el mayor reto y tarea pendiente en la agenda de la actividad mercadológica. Ya que, si cada ser humano pretende vivir medianamente en bienestar con lo que queda de los recursos sostenibles del planeta, se debe tomar conciencia de que se debe evitar el derroche, a pesar que la economía actual esté basada, en cierta medida, en el derroche y el consumismo. Esto puede y debe cambiar. ¿Las empresas lo tomarán como tarea realmente urgente? Quizás, pasar a este nivel superior, sea la prueba de fuego para las corporaciones, a través de la cual, se medirá la sinceridad y honestidad de las campañas que tocan temas medioambientales y de responsabilidad social corporativa que a la fecha, están siempre ligadas a algún tipo de ganancia o rédito económico, social o político.

\section{Conclusiones}

El marketing, al ser una actividad de carácter social, impacta sobre la sociedad y el entorno cultural. Este impacto puede ser positivo o negativo. Las empresas deben promover cambios positivos sobre las sociedades con las que interactúan, a través de programas de responsabilidad social bajo un marco de actuación ética.

Las empresas deben generar inclusión social, cambio cultural positivo que impacte, a su vez, en otros aspectos relacionados, tanto con le empresa como con la sociedad en general, dentro de un marco de ética y responsabilidad 
social claramente demarcado. Todo cambio social o cultural debe generar progreso, tanto en lo referente a calidad de vida, como a nivel de vida. Asimismo, el marketing debe constituirse en herramienta activa propulsora del cambio social y cultural. El mayor reto de las corporaciones, será realmente lograr que el consumo se reduzca, pero .... ilas grandes corporaciones querrán tomar el desafío? Consideramos enfáticamente que deberán hacerlo en pro de una auténtica responsabilidad social corporativa.

\section{Referencias}

Angulo, N. (2010). Pobreza, medio ambiente y desarrollo sostenible. "Nómadas. Revista Crítica de Ciencias Sociales y Jurídicas", 26, 33-42.

Arellano, R. (2001). “Estilos de vida en el Perú. Cómo sienten y piensan los peruanos del siglo XXI". Lima: Arellano Marketing.

DEMUS (Estudio para la Defensa de los Derechos de la Mujer). (2008). "Justicia de género. Indecopi y publicidad sexista”. Lima: DEMUS.

García, J. (200o). El marketing con causa, entre la oportunidad estratégica y la responsabilidad social. "Revista de Fomento Social”, 217, 105-112.

Instituto Nacional de Estadística e Informática (INEI). (2013). "Producto bruto interno por departamentos, 2001 - 2012”. (Documento en formato pdf). Recuperado el 11 de diciembre de 2015 de: https://www.inei.gob.pe/media/MenuRecursivo/publicaciones_digitales/Est/ Lib1104/libro.pdf
Kotler, P.; Gertner, D.; Rein, I. \& Haider, D. (2007). "Marketing internacional de lugares y destinos: Estrategias para la atracción de clientes y negocios en Latinoamérica”. México: Pearson Educación.

Kotler, P. \& Armstrong, G. (2013). "Fundamentos de Mercadotecnia”. México: Pearson Prentice Hall.

López, S. \& Puente, R. (2007). El cliente que reclama es el que más te quiere. "Debates IESA", 12, 52-59.

Malhotra, N. (2008). "Investigación de Mercados". México: Pearson Educación.

Pérez, L. (2004). "Marketing social, Teoría y práctica”. México: Pearson Educación.

Observatorio Socio Económico Laboral del Ministerio de trabajo (MINTRA). (2013). "Diagnóstico socio económico laboral de la Región Arequipa”. (Documento en formato pdf). Recuperado el 11 de diciembre del 2015 de: http:// www.mintra.gob.pe/archivos/file/ estadisticas/peel/osel/2013/Arequipa/ Diagnostico/Diagnostico_012013_ OSEL_Arequipa.pdf

Schiffman, L. (2010). "Comportamiento del consumidor". México: Pearson Educación.

Valdez, R. (2014). Ética mercadológica en un marco de responsabilidad social corporativa. "Illustro", 5, 51-59.

Recibido: 30-09-2015 Aceptado: 12-12-2015 